\title{
Interpretación del test del reloj en una usuaria con enfermedad de Parkinson
}

\section{Interpretation of clock drawing test at user with Parkinson's disease}

\author{
Exequiel Guevara ${ }^{1,2, a}$, Víctor Alarcón ${ }^{3, b}$ \\ ${ }^{1}$ Carrera de Fonoaudiologia, Departamento de Especialidades Médicas, Universidad de La Frontera. Temuco, Chile. \\ ${ }^{2}$ Centro de Capacitación e Investigación en Neurociencias. Temuco, Chile. \\ ${ }^{3}$ Escuela de Psicología, Facultad de Ciencias Sociales y Comunicaciones, Universidad Santo Tomás. Temuco, Chile. \\ ${ }^{a}$ Fonoaudiólogo, magister en neuropsicología. ORCID: https://orcid. org/0000-0001-7250-7319 \\ ${ }^{\mathrm{b}}$ Psicólogo, magister en neuropsicologia. ORCID: https://orcid.org/0000-0002-7537-6585
}

\begin{abstract}
Correspondencia:
Exequiel Guevara

exequiel.gd@gmail.com
\end{abstract}

Recibido: 1 de octubre 2020

Aprobado: 15 de diciembre 2020

Publicación en línea: 30 de diciembre 2020

Conflictos de interés: Los autores declaran no tener conflictos de interés.

Fuente de financiamiento:

Autofinanciado

Citar como: Guevara E, Alarcón V. Interpretación del test del reloj en una usuaria con enfermedad de Parkinson. An Fac med. 2020;81(3):372-3 DOI: https://doi.org/10.15381/anales. v81i3.18858.

\section{An Fac med. 2020;81(3):372-3. / DOI: https://doi.org/10.15381/anales.v81i3.18858}

\section{Sr. Editor,}

La enfermedad de Parkinson (EP) es la segunda enfermedad neurodegenerativa en el mundo, superada únicamente por la enfermedad de Alzheimer (EA) ${ }^{(1)}$. Se caracteriza por la presencia de síntomas motores y no motores, encontrándose dentro de estos últimos la afectación cognitiva. Estas alteraciones cognitivas pueden adquirir distintas manifestaciones, las que pueden ir desde una leve disminución en las funciones ejecutivas (perfil cognitivo de EP normal), pasando por la presencia de un síndrome disejecutivo acompañado de alteraciones visuoespaciales, déficits atencionales y enlentecimiento en la velocidad de procesamiento (perfil cognitivo de EP con deterioro cognitivo) ${ }^{(2)}$, e incluso avanzar hacia una demencia asociada a la EP, la cual se presenta con una grave alteración disejecutiva, empeorando además todos los otros síntomas mencionados con anterioridad, y comprometiendo la funcionalidad, conducta, emoción y sensopercepción ${ }^{(3)}$.

Reportamos el caso de una mujer de 73 años, jubilada, con 5 años de escolaridad y con un diagnóstico de EP de 13 años de evolución, que acude a evaluación neuropsicológica por iniciativa propia. Durante la evaluación, se evidenciaron puntajes globales dentro de la normalidad para el resto de las pruebas cognitivas efectuadas, entre las que se encuentran el minimental, el test de las fotos, el frontal assessment batery (FAB), aunque fallando en las tareas de control inhibitorio, y el Pfeiffer de memoria. Sin embargo, destaca lo realizado por la usuaria al efectuar el test del reloj a la orden (TRO), el cual consiste en pedirle al usuario que realice una circunferencia lo más grande posible, luego se le solicita que ponga todos los números dentro de la misma y finalmente se le menciona que debe poner la hora once y diez. Posteriormente, se consigna puntaje por la circunferencia (2 puntos), por los números (4 puntos) y por las manecillas del reloj (4 puntos) ${ }^{(4)}$.

La usuaria, una vez entregadas las instrucciones verbales, con dificultades intentó realizar una circunferencia, no logrando su objetivo a cabalidad. A continuación, al comenzar a dibujar los números dentro de la figura, no ubicó al 12 en su posición ni los siguientes números. Es más, los situó a todos dentro de lo que se podría considerar la mitad derecha, e incluso agregó un número 13, en donde ella se percata que algo no estuvo bien, pero no logró identificar sus errores. Posteriormente, al momento de colocar la hora, ubicó un "110" en el centro, de lo que se desprende que sería las 11:10 (figura 1), obteniendo una puntua- 
ción final de 2 puntos. Se considera como puntaje de corte un total de 6 puntos o menos para estar en presencia de algún deterioro cognitivo ${ }^{(4)}$.

De lo anteriormente descrito, y considerando las dificultades cognitivas que pudiese presentar debido a la EP, se desprende que es muy probable que a raíz de sus déficits atencionales haya presentado dificultades en la retención de la instrucción verbal. Asimismo, es posible que haya comenzado a experimentar fallas en sus funciones ejecutivas, principalmente en las tareas de planificación, de inhibición, en la memoria de trabajo, de ir a buscar la información mnésica al- macenada y en la velocidad de procesamiento, todos procesos necesarios para poder evocar correctamente el recuerdo y posterior ejecución del reloj. Del mismo modo, tanto en el dibujo de la circunferencia como de los números, se observaría un presunto déficit visuoespacial, lo que sería totalmente factible de encontrar en personas con EP sin demencia ${ }^{(5)}$.

Por tanto, la recomendación es no centrarse netamente en los puntajes arrojados por las diferentes pruebas, sino ser más curiosos y auto-exigentes en la interpretación de las mismas. De igual manera, y si bien es importante considerar el perfil cognitivo y la progresión de la enfermedad en las personas con EP, no se puede olvidar que también el nivel educativo cumple un rol importante en las manifestaciones clínicas, existiendo evidencias que los diferentes instrumentos de evaluación neuropsicológica, incluido el test del reloj, pueden ser influenciados significativamente por esta variable ${ }^{(6)}$.

Finalmente, se invita a los clínicos y académicos a ampliar la mirada en torno a la evaluación neuropsicológica, en donde resulta muy importante contar con variados instrumentos que permitan evaluar la mayor cantidad de funciones cognitivas.

\section{REFERENCIAS BIBLIOGRÁFICAS}

1. Perea M, Ladera V. Neuropsicología de la Enfermedad de Parkinson. Revista Neuropsicología, Neuropsiquiatría y Neurociencias. 2012;12(1): 219-241.

2. Marras C, Tröster A, Kulisevsky J, Stebbins G. The Tools of the Trade: A State of the Art "How to Assess Cognition" in Patient With Parkinson's Disease. Movement Disorders. 2014;29(5): 584-596. DOI: https://doi.org/10.1002/mds.25874

3. Caballol N, Martí MJ, Tolosa E. Cognitive dysfunction and dementia in Parkinson disease. Movement Disorders. 2007; 22(S17): S358-S366. DOI: https:// doi.org/10.1002/mds. 21677

4. Cacho J, Garcia-Garcia R, Arcaya L, Vicente J, Lantada N. Una propuesta de aplicación y puntuación del test del reloj en la enfermedad de Alzheimer. Rev Neurol. 1999;28(7): 648-655. DOI: https://doi. org/10.33588/rn.2807.98501

5. Curtis AF, Masellis M, Camicioli R, Davidson $H$, Tierney MC. Cognitive profile of non-demented Parkinson's disease: Meta-analysis of domain and sex-specific deficits. Parkinsonism and Related Disorders. 2019;60:32-42. DOI: https://doi. org/10.1016/j.parkreldis.2018.10.014

6. Kim H, Chey J. Effects of education, literacy, and dementia on the Clock Drawing Test performance. Journal of the International Neuropsychological Society. 2010; 16(6): 1138-1146. DOI: https:// doi.org/10.1017/S1355617710000731

Figura 1. Test de reloj en paciente con enfermedad de Parkinson. 